\title{
Pillarplexes as molecular functional pores in the solid state
}

\author{
Alexander Pöthiga \\ aTechnische Universität München, Lichtenbergstr. 4, 85748 Garching, Germany \\ alexander.poethig@tum.de
}

Recently, we introduced a new compound class of organometallocavitands (i.e. metallocavitands exhibiting carbon metal bonds), the so-called pillarplexes (Figure 1, A). ${ }^{[1]}$ These multinuclear coinage-metal based complexes, bearing tailor-made macrocyclic NHC ligands (N-heterocyclic carbenes), can act as molecular pores in solution.

The pillarplexes have unique properties as they are intrinsically photoluminescent, they can be easily made water-soluble via anion exchange and they are exclusively shape selective hosts for linear molecules (Figure 1, B). Furthermore, they can also be employed as functional components of mechanically interlocked molecules, namely rotaxanes, to create a $\mathrm{pH}$-dependent molecular switch via reversible acidic decomposition of the cavitand (Figure 1, C). ${ }^{[2]}$ Hereby, the metals can be released at low $\mathrm{pH}$ and taken up again at higher $\mathrm{pH}$.

The transfer of the molecular properties into the solid state by using the pillarplexes as building blocks in crystal engineering paves the way to a novel class of functional porous materials.

A

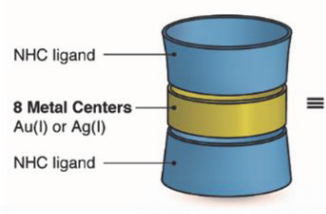

C

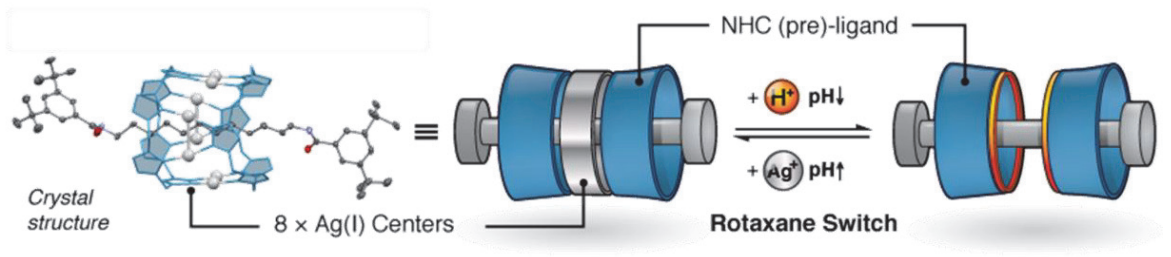

Figure 1. (A) Structure of the organometallic pillarplex cavitand; (B) properties of the gold(I) pillarplex cavitand; (C) pH switchable organometallic [2]rotaxane employing a pillarplex as ring component.

References:

[1] P. J. Altmann, A. Pöthig*, J. Am. Chem. Soc. 2016, 138, 13171-13174.

[2] P. J. Altmann, A. Pöthig*, Angew. Chem., Int. Ed. 2017 129, 15939-15942. 